\title{
404 - Factors associated with diagnostic delay in younger-onset dementia iagnostic delay in younger-onset dementia
}

Samantha M. Loi, Anita M. Goh, DPsych, Dhamidhu Eratne, Ramon Mocellin, Sarah Farrand, Andrew Evans, Charles Malpas, Wendy Kelso, Mark Walterfang, MBBS PhD FRANZCP, Dennis Velakoulis, MBBS,

Background: Younger-onset dementia (YOD) is a dementia of which symptom onset occurs at 65 years or less. There are approximately 27000 people in Australia with a YOD and the causes can range from Alzheimer's dementia (AD), frontotemporal dementia (FTD), metabolic and genetic disorders. It is crucial to obtain a definitive diagnosis as soon as possible in order for appropriate treatment to take place and future planning. Previous research has reported 4-5 years to get a diagnosis (Draper et al. 2016) and factors associated with delay include younger age (van Vliet et al. 2013) and psychiatric comorbidity (Draper et al. 2016). We report on our experience of diagnostic delay.

Methods: This was a retrospective file review of 10 years of inpatients from Neuropsychiatry, Royal Melbourne Hospital, Australia. Neuropsychiatry is a tertiar service which provides assessment of people with cognitive, psychiatric, neurological and behavioural symptoms. Factors such as age of onset, number of services/specialists seen were extracted and analysed using multivariate regression.

Results: Of the 306 individual patients who had a YOD, these were grouped into the major dementia groups (such as AD, FTD, Huntington's disease, vascular dementia, alcohol-related dementia). The most commonly occurring dementia was AD (24.2\%), followed by FTD (23\%). There was an average of 3.7 years $(S D=2.6)$, range 0.5-15 years, of delay to diagnosis. Cognitive impairment, as measured using the Neuropsychiatry Unit Cognitive Assessment (NUCOG) was moderate, with a mean score of 68.9 $(S D=17.9)$. Within the groups of dementia, patients with Niemann-Pick type $C$ (NPC) had the longest delay to diagnosis $F(11,272)=3.677, p<0.0001$, with 6.3 years delay. Age of symptom onset and number of specialists/services seen were the significant predictors of delay to diagnosis $F(7,212)=3.975$, $\mathrm{p}<0.001, \mathrm{R}^{2} 11.6$.

Discussion and conclusions: This was an eclectic group of people with YOD. The results of regression suggests that there are other factors which contribute to the delay, which are not just demographic related. Rarer disorders, such as NPC which present at an early age, and present with symptoms that are not cognitive in nature, can contribute to diagnostic delay.

\section{5 - Emotional Resilience of Older Adults During COVID-19: A Systematic Review of Studies of Stress and Well-Being}

Evelina Sterina BA ${ }^{1}$, Adriana P. Hermida $\mathrm{MD}^{2}$, Danielle J. Gerberi MLIS ${ }^{3}$, Maria I. Lapid MD ${ }^{4}$

Objectives: To examine post-traumatic stress, depression, anxiety, and well-being in older adults under quarantine in the context of epidemics.

Methods: A systematic review of CINAHL, Ovid EBM Reviews, Ovid Embase, Ovid Medline, Ovid PsycINFO, Scopus, and Web of Science databases from 2000-2020 was conducted. Keywords included coronavirus, epidemic, quarantine, stress, mental health, and similar terms. Included studies enrolled

\footnotetext{
${ }^{1}$ Emory University School of Medicine, 100 Woodruff Circle, Suite 231, Atlanta, GA, 30329. Electronic address: evelina.sterina@gmail.com. https://orcid.org/0000-0002-0564-7407

${ }^{2}$ Department of Psychiatry and Behavioral Sciences, Emory University School of Medicine, Atlanta, GA, USA.

${ }^{3}$ Mayo Clinic Library, Mayo Clinic, Rochester, MN, USA.

${ }^{4}$ Department of Psychiatry and Psychology, Mayo Clinic, Rochester, MN, USA.
} 